\title{
DENOISING BY MULTIWAVELET SINGULARITY DETECTION
}

\author{
Charlotte Yuk-Fan $\mathrm{HO}^{1}$, Bingo Wing-Kuen LING ${ }^{2}$ and Peter Kwong-Shun TAM ${ }^{1}$ \\ ${ }^{2}$ Centre for Multimedia Signal Processing \\ ${ }^{1,2}$ Department of Electronic and Information Engineering \\ The Hong Kong Polytechnic University, Hung Hom, Kowloon, Hong Kong \\ Tel: (852)27664094Ｆax: (852)23628439Ｅmail: enhyfan@eie.polyu.edu.hk
}

\begin{abstract}
Wavelet denoising by singularity detection was proposed as an algorithm that combines Mallat and Donoho's denoising approaches. With wavelet transform modulus sum, we can avoid the error and ambiguities of tracing the modulus maxima across scales and the complicated and computationally demanding reconstruction process. We can also avoid the visual artifacts produced by shrinkage. In this paper, we investigate a multiwavelet denoising algorithm based on a modified singularity detection approach. Improved signal denoising results are obtained in comparison to the single wavelet case.
\end{abstract}

\section{INTRODUCTION}

Multiwavelets have been proposed as a generalization of the traditional scalar wavelet, with multiresolution analysis of multiplicity $r$. It has been proposed that multiwavelet bases should be better than wavelet bases for wavelet applications [1]. It is because there are limitations for the single wavelet decomposition. First of all, it cannot offer simultaneous orthogonality, symmetry and compact support together with higher regularity (or approximation order greater than one) [2]. Among the single wavelets only the Haar wavelet fulfils the above properties, but it is discontinuous in the spatial domain. Multiwavelet systems based on two scaling functions and two wavelet functions allow these properties simultaneously. Therefore, the design of multiwavelet filters is more flexible.

Multiwavelet transform has been applied to compression and denoising applications in the past few years. Experimental results [3-6] have shown that multiwavelets generally outperform single wavelets in denoising using thresholding. It is well known that the success of multiwavelets in denoising applications often depends on shorter supports and higher vanishing moments of the multifilters. The number of high amplitude wavelet coefficients created by a brutal transition like an edge is proportional to the width of the supports of the filters. For a more accurate localization of singularities, the number of high amplitude wavelet coefficients produced should be as small as possible. So the supports of the filters should be as short as possible. Moreover, the more the vanishing moments, the smaller the coefficients can be produced over smooth regions at fine scales. Therefore, the multiwavelet coefficients that belong to the noise component can be more easily distinguished at fine scales. The support size increases proportionally to the number of vanishing moments, and multiwavelets can provide a better trade-off of this.

As a result, we extend the denoising algorithm using singularity detection from single wavelet transform to multiwavelet transform. We perform the magnitude sums over the 'cone of influence' and estimate the regularity from their interscale ratios. It is because this was proven to be a better approach to detect singularity in the single wavelet transform [7,8]. Intuitively, the shorter filter supports lead to a shorter 'cone of influence' and better frequency localization. We also investigate the interscale difference, which is complementary to the interscale ratio. Compared to the single wavelet case [7,8], improved results for singularity detection can be achieved, and this is illustrated in the signal denoising results in this paper. The organization of this paper is as the following. First of all, we review the preprocessing and the construction of discrete multiwavelet transform. We then have an understanding of estimating Lipschitz regularity by the multiwavelet transform with preprocessing. We determine the 'cone of influence' and the magnitude sum of the undecimated discrete multiwavelet transform coefficients. Next we describe the denoising algorithm using the multiwavelet singularity detection technique, which is based on thresholding of the multiwavelet coefficients according to their interscale ratio and difference. Finally we present the results and conclusion.

\section{PREPROCESSING}

Preprocessing (or prefiltering) is usually performed before the multiwavelet decomposition. Prefiltering does not exist in the single wavelet transform. It can be thought of as the vectorization of an input signal to produce a certain discrete-time vector-valued signal for the discrete vector-valued wavelet transform. After the multiwavelet reconstruction, postfiltering is applied to give a one-stream recovered signal. Prefiltering often produce correlated coefficients which contain information of the regularity of the input signal. 
Multifiltering captures this information and further characterizes it with its vector-valued transform.

For the singularity detection in the signal denoising application, we experimented two sets of prefilters, the Xia's prefilter [9] and Plonka's prefilter [10]. These prefilters are designed with the constraint that their combination with the multiscaling and multiwavelet filters will have lowpass and highpass properties. Xia's prefilter is orthogonal which is energy preserving, but it is first order only. Plonka's prefilter is symmetric and second order. It is synthesized with perfect reconstruction filters having linear phase. The prefilter coefficient matrices are shown in the Appendix.

\section{CONSTRUCTION OF DISCRETE MULTIWAVELET TRANSFORM}

To construct a one-dimensional compactly supported multiwavelet transform, we begin with two vector functions, the orthonormal multiscaling and multiwavelet vector functions which satisfy the following refinement equations,

$$
\begin{aligned}
& \Phi(t)=2 \sum_{k=0}^{L-1} H_{k} \Phi(2 t-k), \\
& \Psi(t)=2 \sum_{k=0}^{L-1} G_{k} \Phi(2 t-k),
\end{aligned}
$$

where the two matrices $H(\omega)=\sum_{k \in Z} H_{k} e^{-i k \omega}$ and $G(\omega)=\sum_{k \in Z} G_{k} e^{-i k \omega}$ are trigonometric polynomials satisfying $H(\omega) H^{*}(\omega)+H(\omega+\pi) H^{*}(\omega+\pi)=I_{2}$,

$$
H(\omega) G^{*}(\omega)+H(\omega+\pi) G^{*}(\omega+\pi)=O_{2},
$$$$
G(\omega) G^{*}(\omega)+G(\omega+\pi) G^{*}(\omega+\pi)=I_{2},
$$

with real coefficients, where $H^{*}$ denotes the complex conjugate transpose of $H$.

From the above conditions, $\left\{H_{k}\right\}_{0 \leq k \leq L-1}$ and $\left\{G_{k}\right\}_{0 \leq k \leq L-1}$ are $M \times M$ filter matrices which form an orthogonal FIR multifilter bank and so they generate a set of compactly supported scaling and multiwavelet vector functions, $\Phi=\left(\phi_{1}, \phi_{2}\right)^{T}$ and $\Psi=\left(\psi_{1}, \psi_{2}\right)^{T}$, respectively. Let $V_{j}$ be the multiresolution analysis space generated by $\Phi$, that is $V_{j}=\operatorname{span}\left\{2^{j / 2} \phi_{l}\left(2^{j}-k\right)\right\}_{1 \leq l \leq 2, k \in Z}$. As $V_{j+1}=V_{j} \oplus W_{j}, W_{j}$ is the orthogonal complement of $V_{j}$ in $V_{j+1}$, we also have $W_{j}=\operatorname{span}\left\{2^{j / 2} \psi_{l}\left(2^{j}-k\right)\right\}_{1 \leq l \leq 2, k \in Z}$.

For any continuous function $f(t)$ in $V_{o}$, it can be expanded as $f(t)=\sum\left(c_{1, n}^{(0)} \phi_{1}(t-n)+c_{2, n}^{(0)} \phi_{2}(t-n)\right)$. The function $f$ is completely determined by the sequence $\left\{\left(c_{1, n}^{(0)}, c_{2, n}^{(0)}\right)^{T}\right\}$. Let $J$ be the lowest number of decomposition level which is negative. Since $V_{0}=W_{-1} \oplus V_{-1}=\cdots=W_{-1} \oplus \cdots \oplus W_{J} \oplus V_{J} \quad$ and $2^{j / 2} \phi_{l}\left(2^{j}-k\right)$ and $2^{j / 2} \psi_{l}\left(2^{j}-k\right)$ are orthonormal to each other, $f$ can also be expanded as,

$$
f(t)=\sum_{n}\left(\sum_{l=1}^{2} c_{l, n}^{(-1)} \frac{1}{\sqrt{2}} \phi_{l}\left(\frac{t}{2}-n\right)+\sum_{l=1}^{2} d_{l, n}^{(-1)} \frac{1}{\sqrt{2}} \psi_{l}\left(\frac{t}{2}-n\right)\right)
$$

$f(t)=\sum_{n}\left(\sum_{l=1}^{2} c_{l, n}^{(J)} 2^{J / 2} \phi_{l}\left(2^{J} t-n\right)+\sum_{j=J}^{-1} \sum_{l=1}^{2} d_{l, n}^{(j)} 2^{j / 2} \psi_{l}\left(2^{j} t-n\right)\right)$ where $c_{l, k}^{(j)}$ and $d_{l, k}^{(j)} \in \mathfrak{R}$ are given by

$$
\begin{aligned}
& c_{l, k}^{(j)}=\int f(t) 2^{j / 2} \phi_{l}\left(2^{j} t-k\right) d t \\
& d_{l, k}^{(j)}=\int f(t) 2^{j / 2} \psi_{l}\left(2^{j} t-k\right) d t
\end{aligned}
$$

For the discrete-time case, we denote $c_{n}^{(j)}=\left(c_{1, n}^{(j)}, c_{2, n}^{(j)}\right)^{T}$ and $d_{n}^{(j)}=\left(d_{1, n}^{(j)}, d_{2, n}^{(j)}\right)^{T}$, a discrete function $f[n]$ can be expanded as, $f[n]=\sum_{n}\left(c_{n}^{(j)}\right)^{T} 2^{j / 2} \Phi\left(2^{j} t-n\right)$

$=\sum_{n}\left(\left(c_{l, n}^{(j-1)}\right)^{T} 2^{j-1 / 2} \Phi_{l}\left(2^{j-1} t-n\right)+\left(d_{l, n}^{(j-1)}\right)^{T} 2^{j-1 / 2} \Psi_{l}\left(2^{j-1} t-n\right)\right)$

For the analysis bank, we have the decomposition algorithm $\quad c_{k}^{(j-1)}=\sqrt{2} \sum_{n} H_{n-2 k} c_{n}^{(j)}$,

$$
d_{k}^{(j-1)}=\sqrt{2} \sum_{n} G_{n-2 k} c_{n}^{(j)} \text {. }
$$

For the synthesis bank, we have the reconstruction algorithm $c_{k}^{(j)}=\sqrt{2} \sum_{n}\left(H_{k-2 n}^{T} c_{n}^{(j-1)}+G_{k-2 n}^{T} d_{n}^{(j-1)}\right)$.

In this paper, we present the denoising results obtained from using the GHM multifilters [11]. GHM multifilters have many nice properties. The translates of the multiscaling and multiwavelet functions are orthogonal. The multiscaling and multiwavelet functions are quite smooth and they have enough vanishing moments to produce small transform coefficients at fine scales. The scaling functions have short support $[0,1]$ and $[0,2]$ while $[0,3]$ is expected for scalar case with scaling function satisfying a two-scale equation with 4 coefficient matrices. They are also of second order approximation, which can represent constant and linear functions by a linear combination of their translates. They are smoother and have slightly shorter supports than CL multifilters [12]. The multifilter coefficient matrices are shown in the Appendix.

\section{ESTIMATION OF LIPSCHITZ REGULARITY}

From [13], wavelet methods for measuring Lipschitz regularity was introduced. Regularity is measured by looking at the asymptotic decays of wavelet transform coefficients instead of Fourier transform coefficients. This is possible because wavelets can simultaneously localize signals in time and frequency. The property of localization in time enables us to estimate local regularity, whereas the localization in frequency enables the measurement.

Multiwavelets are generated by several scaling functions. Preprocessing is necessary when applying discrete multiwavelet filterbank to scalar signals. The combined filter responses may not provide localization in frequency domain. It can be shown that with an appropiate designed prefilter and postfilter, one can 
estimate the Lipschitz regularity from multiwavelet coefficients.

\section{Multiwavelet Transform Magnitude Sum}

There are many disadvantages of estimating the local Lipschitz exponents of a signal by tracing the evolution of its wavelet transform modulus maxima [7,8]. Therefore, for the multiwavelets case, we estimate the Lipschitz regularity from the interscale evolution of the magnitude sum over the 'cone of influence'. We then perform signal denoising by thresholding the multiwavelet coefficients according to the interscale ratios and differences of the magnitude sums. The 'cone of influence' and the magnitude sum for the multiwavelet transform are determined as the following.

\section{Cone of Influence (COI)}

The COI represents the support length of the wavelet filter. The support length is different at different scales. Let the support of $\psi_{l}$ be $\left[-K_{l}, K_{l}\right]$ for $l=1,2$. The COI of $v$ in the scale-space plane, $C O I_{l, v}^{(j)}$, is the set of index pairs $\left(n, 2^{-j}\right)$ such that $n$ is included in the support of $\psi_{l}$. Since the support of $\psi_{l}$ is equal to $\left|n-2^{-j} v\right|<K_{l} 2^{-j}$, the COI is determined as

$$
C O I_{l, v}^{(j)}=\left\{\left(n, 2^{-j}\right):\left|n-2^{-j} v\right| \leq K_{l} 2^{-j}\right\} .
$$

The COI of $v$ consists of the scale-space points $\left(n, 2^{-j}\right)$ for which the support of $\psi_{l}$ intersects at $n=v$. If $n$ is inside the COI of $v$ then $d_{l, n}^{(j)}$ depends on the value of $f$ in the neighborhood of $v$. Since $|n-v| / 2^{-j}<K_{l}$, the pointwise Lipschitz condition is identical to the uniform Lipschitz condition.

\section{Magnitude Sum over the COI}

Let $N_{l, v}^{(j)}$ be the WTMS at $v$ over the COI of $v$. We have

$$
N_{l, v}^{(j)} f=\sum_{(l, n) \in \operatorname{COI}_{l, v}^{(j)}}\left|d_{l, n}^{(j)}\right| \cdot
$$

Then from the interscale ratio of the magnitude sum, we can characterize and estimate the Lipschitz regularity of a signal.

\section{DENOISING ALGORITHM}

The denoising algorithm is simple and easy to be implemented. The basic procedures are listed as the following:

1. Perform the discrete multiwavelet transform (5 levels of decomposition) after prefiltering the noisy test signal.

2. Determine the COI for all scales and compute the magnitude sum of the transformed coefficients over the COI for all positions.

3. Compute the interscale ratio of the magnitude sum at each position. Retain those multiwavelet coefficients with interscale ratios higher than 2.
4. Reconstruct the signal from the selected multiwavelet coefficients using the inverse discrete multiwavelet transform and postfiltering.

Here are some remarks for the denoising algorithm:

1. As it is observed by the authors in [8] that WTMS of some small irregular signals with Lipschitz exponents between -1 and 0 will also increase as the scale increases. This enables them to falsely fulfill the interscale ratio thresholding criterion. So the interscale difference thresholding criterion was also employed.

2. Two approaches of retaining coefficients were experimented (step 3). They are (i) retaining the coefficients within the COI; (ii) retaining only the coefficients at the positions in the COI which are determined by the maxima of the wavelet transform of an impulse.

3. The suitable threshold values for the interscale ratio is 2 and for the interscale difference is 0.1 for retaining the coefficients within the COI. While they are $\sqrt{2}$ and 0.01 respectively for retaining the impulse maximal coefficients in the COI.

4. Both of the approaches use a joint selection, that is to consider the magnitude sum of the square sum of the magnitude of the two coefficient components.

\section{RESULTS}

As a preliminary work, we experimented the above denoising algorithm with a length-256 1-d noisy signal using the Xia's prefilter and the GHM multifilters. The original signal contains various features such as steps, regular slopes, steep slopes and irregular structures. White Gaussian noise with noise variance equal to 0.1 is added to the original signal to produce a noisy signal with MSE equal to 0.0085 (Figure 1(a)). $30.63 \%$ reduction in MSE was obtained (the MSE decreases from 0.007387 to 0.005124 ) by the previous denoising algorithm using the single wavelet singularity detection [7]. In our experiment, $34.1 \%$ reduction in MSE is obtained (the MSE decreases from 0.0085 to 0.0056 ) by the denoising algorithm using the multiwavelet singularity detection. The reconstructed denoised signal is shown in Figure 1(b).

We also experimented the same original signal corrupted by the white Gaussian noise with noise variance equal to 0.05 and impulsive noise to produce a noisy signal with a MSE equal to 0.0343 (Figure 2(a)). We used the Plonka's prefilter and the GHM multifilters for the multiwavelet decomposition. $77.96 \%$ reduction in MSE was obtained (the MSE decreases from 0.036909 to 0.008133 ) by the previous denoising algorithm using the single wavelet singularity detection [8]. In our experiment, $81.04 \%$ reduction in MSE is obtained (the MSE decreases from 0.0343 to 0.0065 ) by the denoising algorithm using the multiwavelet singularity detection. The reconstructed denoised signal is shown in Figure 2(b). 
We found that retaining those coefficients within the COI is particularly effective for denoising the white Gaussian noise, while retaining those maxima coefficients (remark 2) in the COI is particularly effective for denoising the white Gaussian noise and impulsive noise. Moreover, it was found that performing selection in a joint manner (remark 4) is better than in an individual manner.

\section{CONCLUSION}

In this paper, we extended the singularity detection from the single wavelet transform to the multiwavelet transform. We developed the multiwavelet singularity detection and illustrated it with the signal denoising results. From the preliminary denoising results of a 1-d white Gaussian noise and impulsive noise corrupted signals, we verified the advantageous properties of the multiwavelet transform over the single wavelet transform for singularity detection. We account that the shorter supports of the multifilters contribute to the improvement of the singularity detection, as the size of the 'cone of influence' becomes smaller and so the overlappings of the non-isolated transformed singularities become less. The vanishing moments of the multifilters are also enough to have better signal approximation, and a higher extent of noise attenuation upon thresholding can be obtained. We conclude that signal denoising using the multiwavelet singularity detection technique is better than using the single wavelet singularity detection one.

\section{APPENDIX}

Xia's prefilter:

$Q_{0}=\left(\begin{array}{cc}\sqrt{6}-1 / 2 & \sqrt{6}+1 / 2 \\ \sqrt{3} / 2 & \sqrt{3} / 2\end{array}\right)$

Plonka's prefilter and postfilter:

$Q_{0}=\left(\begin{array}{cc}\sqrt{6} / 6 & \sqrt{6} / 6 \\ \sqrt{3} / 3 & 0\end{array}\right) \quad P_{0}=\left(\begin{array}{cc}0 & 1 \\ -1 / \sqrt{2} & 2\end{array}\right)$

GHM multifilters:

$$
\begin{array}{ll}
H_{0}=\left(\begin{array}{cc}
3 / 10 & 2 \sqrt{2} / 5 \\
-\sqrt{2} / 40 & -3 / 20
\end{array}\right) & H_{1}=\left(\begin{array}{cc}
3 / 10 & 0 \\
9 \sqrt{2} / 40 & 1 / 2
\end{array}\right) \\
H_{2}=\left(\begin{array}{cc}
0 & 0 \\
9 \sqrt{2} / 40 & -3 / 20
\end{array}\right) & H_{3}=\left(\begin{array}{cc}
0 & 0 \\
-\sqrt{2} / 40 & 0
\end{array}\right) \\
G_{0}=\left(\begin{array}{cc}
-\sqrt{2} / 40 & -3 / 20 \\
-1 / 20 & -3 \sqrt{2} / 20
\end{array}\right) & G_{1}=\left(\begin{array}{cc}
9 \sqrt{2} / 40 & -1 / 2 \\
9 / 20 & 0
\end{array}\right) \\
G_{2}=\left(\begin{array}{cc}
9 \sqrt{2} / 40 & -3 / 20 \\
-9 / 20 & 3 \sqrt{2} / 20
\end{array}\right) & G_{3}=\left(\begin{array}{cc}
-\sqrt{2} / 40 & 0 \\
1 / 20 & 0
\end{array}\right)
\end{array}
$$

\section{ACKNOWLEDGEMENT}

The work obtained in this paper was supported by a research grant (project number G-YD26) from the Hong Kong Polytechnic University, and by the Centre for Multimedia Signal Processing, The Hong Kong Polytechnic University.

\section{REFERENCES}

[1] G. Strang and V. Strela, "Short Wavelets and Matrix Dilation Equations," IEEE Trans. on Sig. Proc., Vol. 43, pp. 108-115, Jan. 1995.

[2] I. Daubechies, Ten Lectures on Wavelets, SIAM, Philadelphia, 1992.

[3] V. Strela, P.N. Heller, G. Strang, P. Topiwala and C. Heil, "The Application of Multiwavelet Filterbanks to Image Processing," IEEE Trans. on Sig. Proc., Vol. 8, pp. 548-563, Apr. 1999.

[4] T. R. Downie and B. W. Silverman, "The Discrete Multiple Wavelet Transform and Thresholding Methods," IEEE Trans. on Sig. Proc., Vol. 46, pp. 2558-2561, Sep. 1998.

[5] W. Ling, "Orthogonal Multiwavelets Transform for Image Denoising," Proc. 5th Int. Conf. on Sig. Proc. (WCCCICSP), Vol. 2, pp. 987-991, 2000.

[6] E. Bala and A. Ertuzun, "Applications of multiwavelet techniques for image denoising," Proc. Int. Conf. on Img. Proc., Vol. 3, pp. 581-584, 2002.

[7] T.-C. Hsung and D. P.-K. Lun, "Denoising by singularity rejection," Proc. of Int. Symp. on Circuits and Systems, Vol. 1, pp. 205-208, 1997.

[8] T.-C. Hsung, D. P.-K. Lun and W.-C. Siu, "Denoising by Singularity Detection," IEEE Trans. on Sig. Proc., Vol. 47, pp. 3139-3144, Nov. 1999.

[9] X.-G. Xia, "A New Prefilter Design for Discrete Multiwavelet Transforms," IEEE Trans. on Sig. Proc., Vol. 46, pp. 1558-1570, Jun. 1998.

[10] G. Plonka, "Approximation properties of multiscaling functions: A Fourier approach," Rostocker Mathematische Kolloquium, Vol. 49, pp. 115-126, 1995.

[11] J. S. Geronimo, D. P. Hardin and P. R. Massopust, "Fractal Functions and Wavelet Expansions Based on Several Scaling Functions," J. of Approximation Theory, Vol. 78, pp. 373-401, 1994.

[12] C. K. Chui and J. Lian, "A Study of Orthonormal Multiwavelets," Applied Numerical Mathematics, Vol. 20, pp. 273-298, 1996.

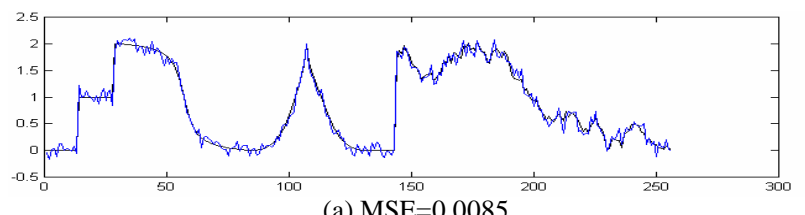

(a) $\mathrm{MSE}=0.0085$
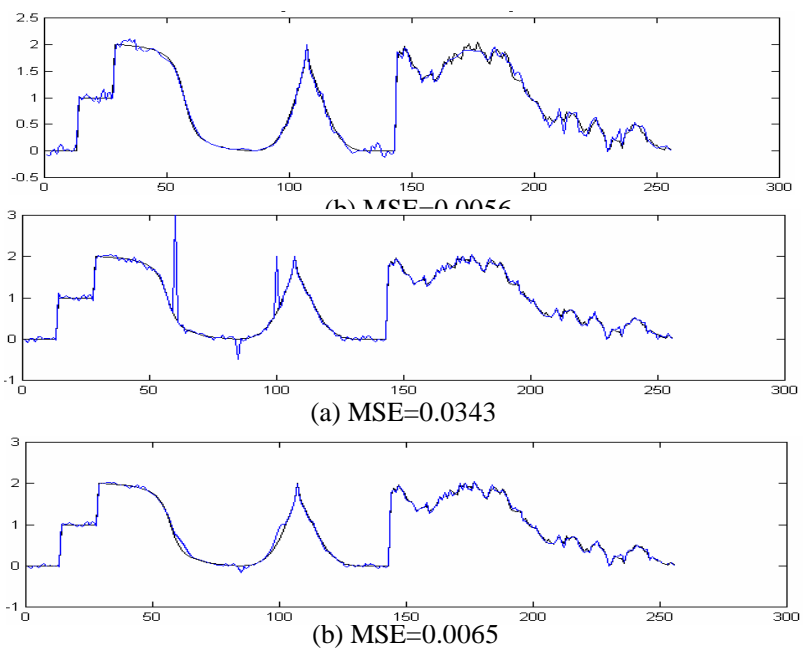

Figure 2 (a) The noisy signal (white Gaussian noise and impulsive noise) (b) the denoised signal by the denoising algorithm using multiwavelet singularity 
[13] S. Mallat and W. L. Hwang, "Singularity detection and processing with wavelets", IEEE Trans on Info. Theory, Vol. 38, pp. 617-643, Mar. 1992. 